\title{
Anomalous origin of the right coronary artery from the left sinus of Valsalva associated with syncope in a young athlete
}

\author{
Steven M. Lilly, MD, Jeffrey M. Schussler, MD, and Robert C. Stoler, MD
}

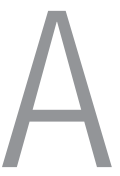

17-year-old African American boy had a syncopal event approximately 30 minutes after playing in a high school football game. He complained of headache just prior to losing consciousness but denied chest pain, dyspnea, or dizziness. His supine blood pressure was 114/69 $\mathrm{mm} \mathrm{Hg}$ without orthostatic variation. The precordial examination was normal with a regular rhythm and no murmur. The electrocardiogram was normal. A transthoracic echocardiogram showed normal ventricular function and chamber sizes but demonstrated a possible abnormal origin of the right coronary artery (RCA). Computed tomographic coronary angiography (CTCA) confirmed that the RCA originated from the left sinus of Valsalva, a finding generally known as anomalous coronary artery from the opposite sinus (ACAOS). The RCA began adjacent to the ostium of the left main coronary artery, coursed between the aorta and pulmonary trunk, and had a characteristic "slit-like" ostial orifice (Figure).

Given this young patient's presentation of exertional syncope in the setting of right ACAOS, surgical correction was performed. The procedure involved "unroofing" the origin of the anomalous RCA and resuspending the aortic valve commissure, involving a total bypass pump time of 92 minutes. The patient recovered well and intended to resume activity in competitive sports.

\section{DISCUSSION}

Among coronary artery anomalies, ACAOS poses a relatively higher risk of sudden death, particularly in the young and when the anomalous artery courses between the ascending aorta and pulmonary trunk $(1,2)$. One registry documented that of 387 sudden deaths among young athletes, $53(13.7 \%)$ were due to coronary anomalies, many of which were ACAOS (3). Most individuals with ACAOS remain asymptomatic, but when symptoms occur they may include sudden cardiac death, dyspnea, angina pectoris, dizziness, palpitations, and syncope (1). The patient presented here experienced a syncopal event following competitive sports, after which a right ACAOS was initially suspected by transthoracic echocardiogram and then confirmed by CTCA.

Right ACAOS is more common than left ACAOS (where the left main coronary artery arises from the right coronary cusp) and is generally considered more benign. Myocardial iscemia and sudden death, however, can be associated with both types of anomalies (4). While it is agreed that surgical correction is the standard of care for left ACAOS when found, the management of right ACAOS is more difficult. Imaging, usually with computed tomography or magnetic resonance imaging, is helpful in defining high-risk features, including the presence of an intramural segment within the aortic wall, the shape and size of the orifice (usually slit-like), and the size of myocardium supplied by the anomalous artery. CTCA in particular is now commonly used to define the characteristics of coronary anomalies (5). The presence of ischemic symptoms may be helpful in defining a high-risk population. Such symptoms are uncommon, particularly in young patients who may present with a single episode of syncope or sudden cardiac death. Stress testing can also be useful to objectively demonstrate myocardial ischemia if the management strategy remains unclear.

Treatment options for these patients include observation, percutaneous intervention (stenting), or surgery. An observational approach would typically include restriction from further competitive sports. Stenting can be technically difficult, with unclear longterm success rates, and currently is done in relatively few centers.

Traditional cardiac surgery for such coronary anomalies includes coronary bypass; corrective procedures aimed at reducing the likelihood of ischemia from the anomalous vessel have become a preferred strategy. Corrective procedures are particularly used in young patients without obstructive coronary artery disease, given the concern of graft longevity over decades and competitive flow in the native coronary arteries that may increase the rate of graft failure (6). Corrective surgical procedures include direct implantation of the anomalous artery, unroofing the intramural segment of the vessel within the aortic wall, and/or osteoplasty, which aims to create a new ostium at the end of the intramural segment as the artery branches away from the aorta. The preferred procedure is highly surgeon and institution dependent, with various case series reporting success rates with each of these techniques $(7,8)$.

From the Division of Cardiology, Department of Internal Medicine, Baylor Jack and Jane Hamilton Heart and Vascular Hospital and Baylor University Medical Center at Dallas.

Corresponding author: Robert C. Stoler, MD, Director, Cardiac Catheterization Laboratory, Baylor Jack and Jane Hamilton Heart and Vascular Hospital and Baylor University Medical Center at Dallas, 621 North Hall Street, Suite 400, Dallas, Texas 75226 (e-mail: rstoler@cctheart.com). 

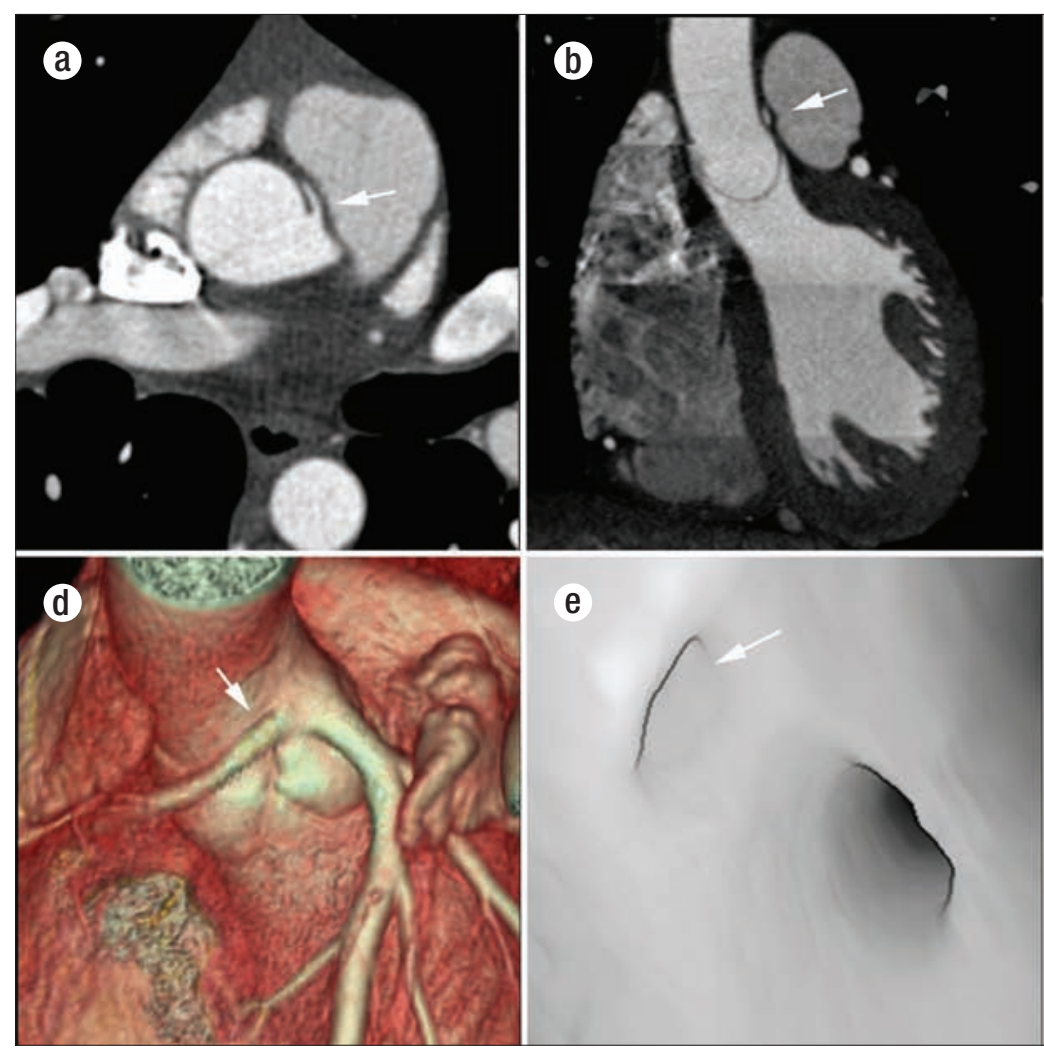

e

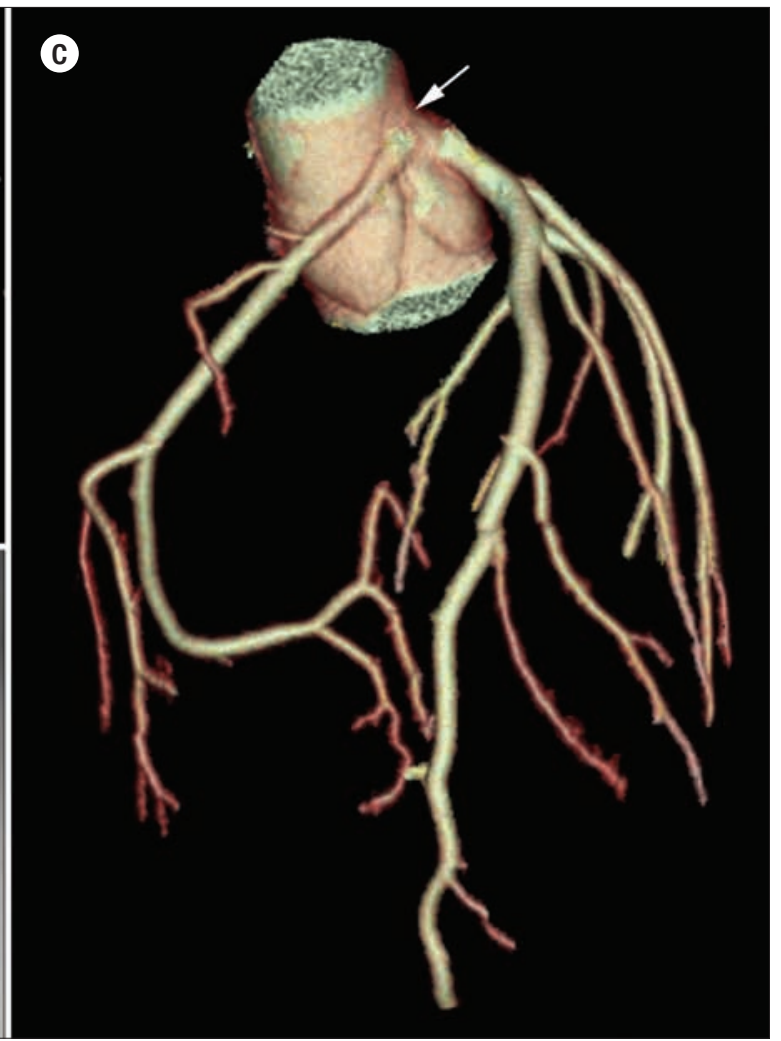

Figure. Computed tomographic coronary angiography images of this patient with an anomalous right coronary artery (RCA) from the opposite sinus. (a, b) Images showing the origin of the RCA from the left sinus of Valsalva as it takes an interarterial course between the aortic and pulmonary trunks (arrows). (c, d) Three-dimensional reconstructions of the coronary anatomy showing the full course of the RCA in relation to the left main and left anterior descending arteries (arrows). (e) A navigator view that shows the slit-like ostium of the anomalous RCA and its close proximity to the ostium of the left main artery.

In the case presented here, an unroofing procedure was chosen, which is aimed at relieving systolic compression of the proximal anomalous artery. Many surgeons and centers also believe unroofing is easier to perform than direct coronary implantation, while the long-term complication rate may be lower (9). In older patients or in those whose anomalies are found incidentally, it is more likely that bypass would be considered if obstructive coronary disease were present; otherwise an observational approach may be advised.

1. Angelini P. Coronary artery anomalies: an entity in search of an identity. Circulation 2007;115(10):1296-1305.

2. Kragel AH, Roberts WC. Anomalous origin of either the right or left main coronary artery from the aorta with subsequent coursing between aorta and pulmonary trunk: analysis of 32 necropsy cases. Am J Cardiol 1988;62(10 Pt 1):771-777.

3. Maron BJ. Sudden death in young athletes. N Engl J Med 2003; 349(11):1064-1075.
4. Ho JS, Strickman NE. Anomalous origin of the right coronary artery from the left coronary sinus: case report and literature review. Tex Heart Inst J 2002;29(1):37-39.

5. Berbarie RF, Dockery WD, Johnson KB, Rosenthal RL, Stoler RC, Schussler JM. Use of multislice computed tomographic coronary angiography for the diagnosis of anomalous coronary arteries. Am J Cardiol 2006;98(3):402-406.

6. Bhimaraj A, Perez-Tamayo A, Pelaez V. Anomalous origin of the right coronary artery in an asymptomatic athlete: what if he did not have a murmur? Cardiology Review 2008;20:47-49.

7. García-Rinaldi R, Sosa J, Olmeda S, Cruz H, Carballido J, Quintana C. Surgical treatment of right coronary arteries with anomalous origin and slit ostium. Ann Thorac Surg 2004;77(5):1525-1529.

8. Erez E, Tam VK, Doublin NA, Stakes J. Anomalous coronary artery with aortic origin and course between the great arteries: improved diagnosis, anatomic findings, and surgical treatment. Ann Thorac Surg 2006;82(3):973-977.

9. Davies JE, Burkhart HM, Dearani JA, Suri RM, Phillips SD, Warnes CA, Sundt TM 3rd, Schaff HV. Surgical management of anomalous aortic origin of a coronary artery. Ann Thorac Surg 2009;88(3):844-847. 